\title{
Modalmetric Fiber Optic Sensor for Security of Collections
}

\author{
M. ŻYCZKOWSKI*
}

Institute of Optoelectronics, Military University of Technology, S. Kaliskiego 2, 00-908 Warszawa 49, Poland

The main aim of the work is the integration of modalmetric sensor in one arm. The proposed sensor is a classical fiber optic cable whose measuring arm is upgraded with multimode sensor. The aim of the research is to assess the impact of modal changes for detection of mechanical disturbances and comparative assessment of detection capabilities of interferometer sensor, modalmetric sensor, and hybrid sensor for different types of excitations. The measurements concerns the area of fundamental research. The results can be used in the construction of sensors with corresponding characteristics and properties, especially to protect museum collections.

DOI: 10.12693/APhysPolA.124.428

PACS: 42.81.Pa, 87.57.-s, 42.79.Qx

\section{Introduction}

Due to the simple construction of modalmetric sensor, the implementation is not complicated. However, the configuration based on fiber loop can cause many difficulties during the implementation process $[1,2]$. Therefore, author presents the integrated sensor in a single arm thus the sensing structure consists of one arm ended with a mirror. This approach is resistant to the problems associated to managing the fiber loop back to the sensor. In addition, this solution reduces the required length of the multimode (MM) fiber. Furthermore, in this configuration, the radiation in the measurement branch is affected by the same disorder twice. Author focuses special attention on the possibility of using this type of sensor system in practice, where finally the best chance of implementations is possessed by a product that meets the minimum requirements of detection and with the lowest cost of implementation. The article presents the results of research on the modalmetric sensor frequency response in the frequency range required for the detection of physical disorder acting on the fiber. Article describes influence of different components of the system such as light source and used fiber mirrors on the frequency response of the sensor. These studies are intended to selecting the best and also the cheapest components for which the system keeps its detection properties with the easiest way to build the system which allows easy implementation in terms of the protection of museum collections.

\section{Principle of operation and construction of the sensor}

Modalmetric sensor presented in Fig. 1 consists of a light source (1). The radiation from source is introduced into the multimode fiber by approximately $280 \mathrm{~m}$ of single-mode (SM) fiber (2), circulator (3) and MM fiber (4) (a measurement arm), MM fiber with sputtering layer of gold mirror (5) and a detector (7) connected to the circulator output of radiation by the approximately $1 \mathrm{~m}$ of optical fibers (6).

*e-mail: mzyczkowski@wat.edu.pl

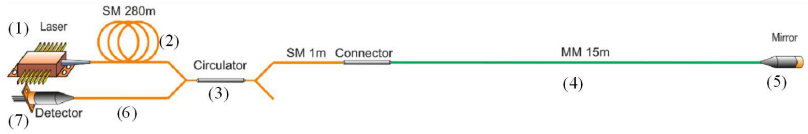

Fig. 1. Scheme of one-arm modalmetric fiber sensor.

Operation of modalmetric sensor [3] is based on a change in the distribution of modes in the MM fiber due to the external disturbances [4]. Coherent light is introduced into the MM fiber with startup SM fiber. During the transmission of light from SM fiber to MM fiber, significant number of modes is excited. Excited modes propagate in the MM fiber, and at the end of it the speckle pattern is formed. External fiber disorder causes changes in polarization, phase, or modes distribution, which changes the speckle pattern at the end of fiber. Due to the interaction of propagating modes in the MM fiber, modalmetric sensor can be considered as interference multibeam sensor $[5,6]$ enclosed in a single fiber. The relationships between the modes are the stochastically stable distribution of intensity of the radiation at the fiber output. According to Goodman's paper [7], the distribution may be represented by Eq. (1) where $\nu$ is optical frequency, and $A(x, y, z)$ is the amplitude of the complete vector

$$
u(x, y, z ; t)=A(x, y, z) \exp (\mathrm{i} 2 \pi \nu t) .
$$

Goodman made an assumption that field incident at $(x, y, z)$ is ideally polarized and perfectly monochromatic. Total amplitude of the field in position $(x, y, z)$ can be represented as the sum of the components due to core areas of scattering on an uneven surface. The amplitude of these vectors can be represented by Eq. (2), where $\left|a_{k}\right|$ and $\phi_{k}$ represents amplitude and phase of the $k$-th scattering area, and $N$ describes the number. The observed amount of radiation in the $(x, y, z)$ can be represented by Eq. (3):

$$
\begin{aligned}
& A(x, y, z)=\sum_{k=1}^{N}\left|a_{k}\right| \exp \left(\mathrm{i} \phi_{k}\right), \\
& I(x, y, z)=\lim _{T \rightarrow \infty} \frac{1}{T} \int_{-T / 2}^{T / 2}|u(x, y, z ; t)|^{2} \mathrm{~d} t \\
& \quad=|A(x, y, z)|^{2} .
\end{aligned}
$$


If the time difference of the various modes propagating in the MM fiber is less than the coherence time of the source of radiation at the output of the MM fiber, one can observe the interference pattern called speckle. In order to increase the amount of information that can be read from speckle pattern, it is desired to increase the contrast described in Eq. (4), where $I_{\max }$ and $I_{\min }$ is the maximum and minimum intensity of the radiation

$$
V=\frac{I_{\max }-I_{\min }}{I_{\max }+I_{\min }}
$$

The contrast is dependent on the source parameters (spectral radiation bandwidth $\Delta f_{s}$, mode spacing $\Delta f_{m}$, modal half width $\delta f$ ) of radiation used in the sensor system and the parameters of the transmission fiber (length $L$, dispersion $D_{m}$ ). Therefore it is important to select the appropriate parameters of fiber and a source. The aim is to achieve small number of the narrow laser lines, and high correlation of the fiber band.

Observation of modalmetric changes in the speckle pattern is the method of detection of the disorder in the sensor. This can be achieved by using a physical limitation of observation area, or a detector analyzing selected area of sample. The presented configuration uses fiber transition between MM and SM fiber as a physical limiter of the field of view of detector. Any change of the speckle pattern is seen on the detector as a change in the intensity of the radiation.

\section{Experimental details and results}

The sensor construction consists of standard telecommunications equipment such as single-mode fiber with $9 \mu \mathrm{m}$ core and $125 \mu \mathrm{m}$ clad, multimode fiber with $62.5 \mu \mathrm{m}$ core and $125 \mu \mathrm{m}$ clad, three-arm single-mode fiber optic circulator. Light source used in the configuration is a laser-C5-1310 Series of multi-quantum well (MQW) distributed feedback (DFB), multimode laser (LD) and SLED diode ESL1310-2111 type Exalos on a standard telecommunications diode, connected to Yokogawa DLM 2054 oscilloscope. Reference system for generating disorders is constructed as a mechanical modulator controlled by the generator Tektronix AFG3021. Image of the modulator head is presented in Fig. 2.

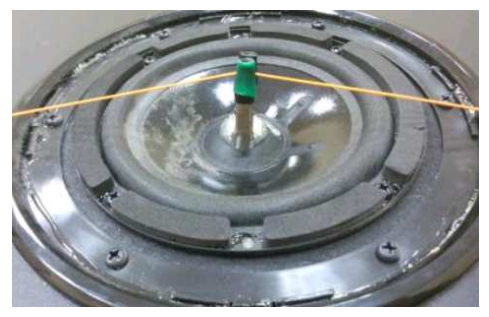

Fig. 2. Photo of the head of mechanical disorders modulator.

The aim of the tests of the modalmetric fiber sensor was to plot the amplitude frequency characteristics by measuring the amplitude of the sensor response to mechanical disturbance generated by modulator with known and constant amplitude and various frequency.
In the first part of research, the system was tested with different kind of reflections of radiation from the end of multimode fiber optic sensor. For the research, self-made mirrors made by sputtering the layer of gold on properly prepared, single or multimode fiber forehead as well as commercially available Faraday rotator mirror with pigtailed single mode fiber were used.

Moreover, the scope of the tests contained also using different types of light sources. Fiber optic sensor was sourced by radiation from semiconductor laser diode with characteristics: strictly single-mode (DFB), multimode (typical LD) as well as wide spectral (SLED). For all types of laser diodes, the characteristics of response on the disorder with the direct change of the laser power as well as through using series of attenuators $2,3,5,10 \mathrm{~dB}$ at the input of the sensor were measured.

According to the present knowledge, it was expected that one of the optical parameter is likely to affect the response of the sensor (sensor characteristics) and is determining a way of the modes propagation in the optical fiber. It was expected that the elimination of cladding modes from optical source can stabilize the system performance, thus it was able to obtain stable and reproducible distribution of modes in the sensor in a steady state. For the test purposes, typical telecommunications mode scrambler was applied. It was made as a section of five single-mode fiber coils on the sleeve with a diameter of a few millimeters.

Disorder signals of fiber-optic interferometric sensors are characterized by that they are involved in the transfer function of the interferometer and they are not directly identifiable as "microphone" signals. In order to have a continuous monitoring of the cable/optical fiber, i.e. hear undistorted vibrations, it would be required to prepare a signal demodulation process. Tests confirm that the optical interference sensors can be used as very sensitive microphone, and they can recognize human speech. In addition, the frequency response range of this type of system is practically unlimited up to the frequency of mechanical range (from single $\mathrm{Hz}$ to $25-30 \mathrm{kHz}$ ). Additionally, it was observed during the work on security sensors that the transmitted changes associated with acoustic/ mechanical fiber disorders during its touching are contained typically in the frequency range up to $500 \mathrm{~Hz}$ and during the few - a few hundred milliseconds.

Due to the fact that the frequency range, interesting from the point of view of the detection of mechanical disorders, is in the range of up to $500 \mathrm{~Hz}$, interference sensor systems do not fully exploit their potential. In addition, the interference sensors generate a response included in the transfer function. In the case of the modalmetric sensor system, amplitude response is a direct reflection of external disturbances, as presented in Fig. 3.

The application of the sensor indicates the detection of mechanical changes with a frequency similar to the acoustic impact of the environment on fiber-optic sensor. Because of that the system was tested only in the frequency range of interest $1-500 \mathrm{~Hz}$. In addition, based 

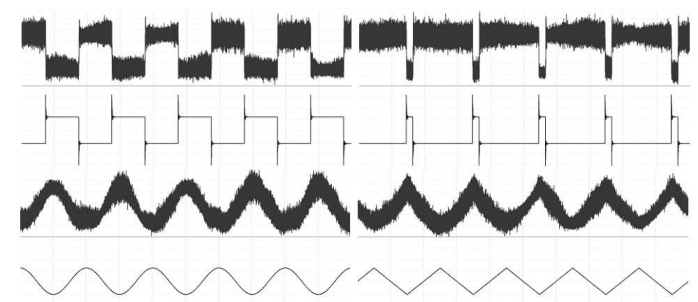

Fig. 3. Sample waveforms of modalmetric fiber sensor (upper wave form) response to the harmonic disturbances.

on the principles of operation, it is expected to receive more linear characteristics than of the fiber-optic interferometer systems and more limited in terms of sensitivity in transmission range. It was assumed that the proper results of a sensor for museum protection should be less sensitive to acoustic vibrations and more suitable for the detection of mechanical disturbances.

The first stage of the study was to measure the system response to mechanical disturbances by changing the end of multimode fiber sensor with various types of mirrors. For this purpose the characteristics of the modalmetric sensor were measured for the three various types of mirrors: sputtering layer of gold on multimode fiber forehead, sputtering layer of gold on single mode fiber forehead, Faraday rotator mirror with pigtailed single mode fiber. Figure 4 presents sensitivity characteristics of the modalmetric sensor system as function of frequency in the range 1 to $500 \mathrm{~Hz}$ for different end of multimode fiber.

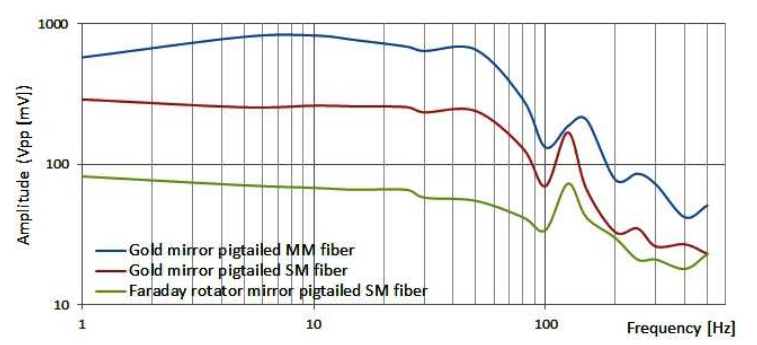

Fig. 4. Characteristics of sensitivity as a function of frequency for different mirrors for the DFB laser $1310 \mathrm{~nm} 1 \mathrm{~mW}$.

As presented on the characteristics in Fig. 4 modalmetric sensor system achieves the best results for the mirror made by sputtering the layer of gold directly into the end of the multimode fiber. As expected, in the configuration with no light transmission from the end of the multimode fiber to a single-mode fiber, the entire range of modal speckle pattern is practically lossless by reflected on multimode fiber, this insures the proper functioning of the sensor system. Therefore, the main conclusion is that for the development of modalmetric sensor it should be created a dedicated fiber optic with passive component (it is not widely popular on the market), in the form of mirror on the multimode fiber. Based on these results, for the next tests, only modalmetric sensor with multimode fiber mirror was used in order to reduce the additional losses in the fiber optic circuit.

The next stage of the research was testing the response of the fiber optic modalmetric sensor in the frequency range of $1-500 \mathrm{~Hz}$ for different levels of optical power in the fiber circuit. For this purpose, the measurements of sensitivity characteristic were performed using a single-mode laser, for which it was possible to continuously adjust the laser power by changing the current power of a laser diode. The measurement results are presented in Fig. 5.

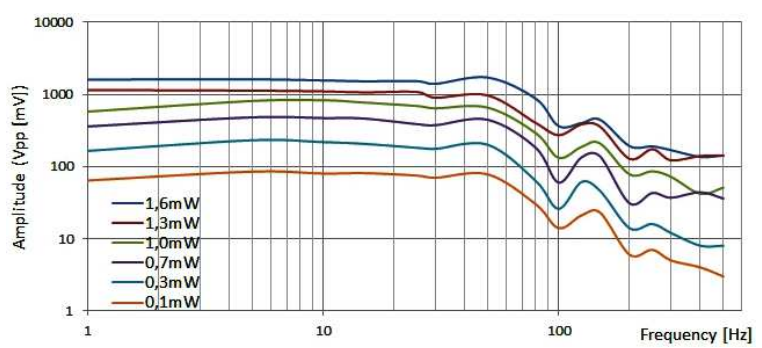

Fig. 5. Characteristics of sensitivity as a function of frequency for various output optical power of DFB laser $1310 \mathrm{~nm}$.

Measurements of characteristics were repeated for the same system conditions, with modification of the limit of the output optical power of laser. For this purpose, a series of attenuators $1.85 \mathrm{~dB}, 3.05 \mathrm{~dB}, 5.11 \mathrm{~dB}$ and $9.87 \mathrm{~dB}$ were used. Output power of the laser was set to maximum $1.81 \mathrm{~mW}$. Sensitivity characteristics of the system with laser output optical power control using optical attenuators are presented in Fig. 6.

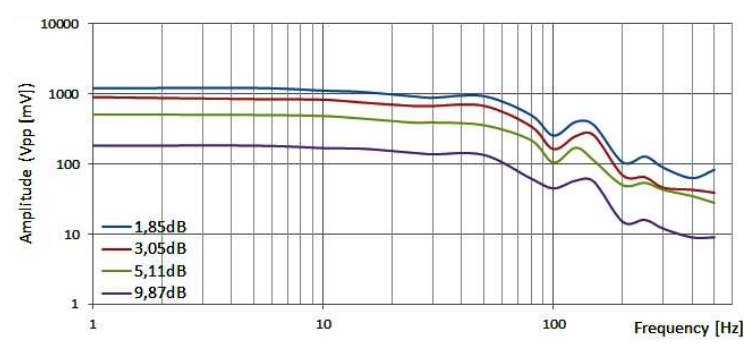

Fig. 6. Characteristics of sensitivity as a function of frequency for the DFB laser $1310 \mathrm{~nm} 1.81 \mathrm{~mW}$ adjustable output optical power with attenuator.

Analyzing the above characteristics, one can conclude that the sensitivity of the modalmetric sensor depends on the level of the optical power supplying the fiber sensor circuit, while the shape of the sensitivity function of frequency remains unchanged for all tested levels of optical power.

According to the conclusions presented above, sensitivity characteristics of the fiber-optic modalmetric sensor for different types of light sources were measured. Light sources used for the measurements represent the family of most common types of optical power supplies for fiber systems in these spectral distribution. Spectral characteristics of used sources are presented in Fig. 7. Spectral 


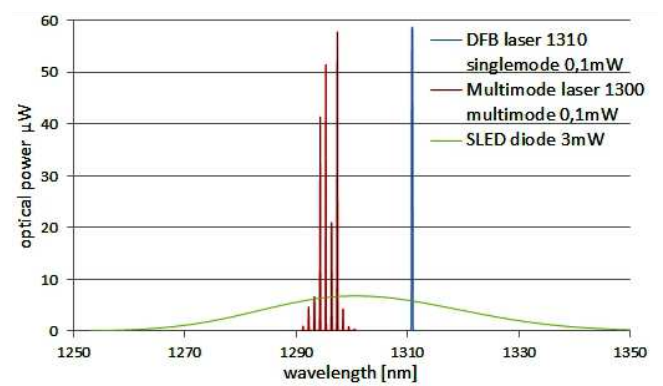

Fig. 7. Spectral characteristics of used light sources.

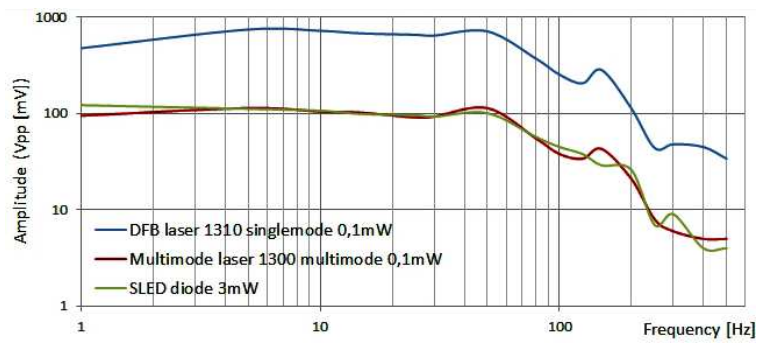

Fig. 8. Characteristics of sensitivity as a function of frequency for different light sources.

range of used sources is within the range from $1250 \mathrm{~nm}$ to $1350 \mathrm{~nm}$.

Results of the measurement of sensitivity characteristics as a function of frequency for three different light sources are presented in Fig. 8.

Based on characteristics presented in Fig. 8, it could be concluded that the response of modalmetric sensor for various optical power suppliers remains unchanged. When supplying the circuit with a single-mode DFB laser, the similar level of the multimode laser power is achieved with higher sensitivity of the modalmetric fiber sensor.

The next stage of the research was to measure the impact of modes scrambler. Modes scrambler was made by winding 5 rolls of fiber on the rod with a diameter 1.25 inches and the 1.25 inches length. According to the theory, the aim of using the modes scrambler is to achieve a uniform distribution of transverse modes in the fiber. Measurement results of sensitivity characteristics as a function of frequency for the three different light sources using a modes scrambler are presented in Fig. 9.

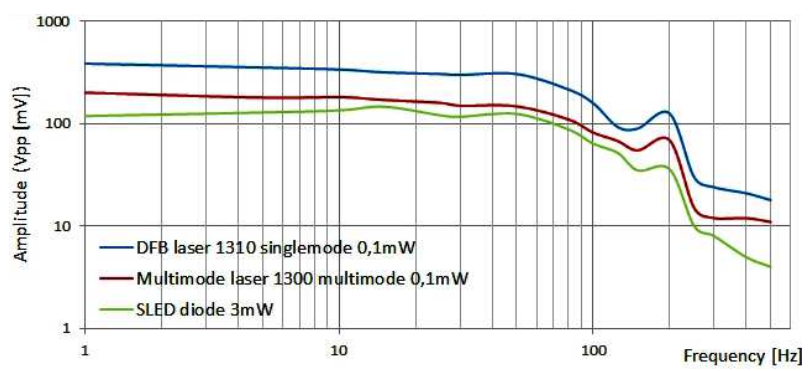

As presented in Fig. 9 using the mode scrambler causes a slight change in characteristics. In the lower range of frequencies, it causes that the system response is more linear than without the modes scrambler. For higher frequency, the modes scrambler causes a slight increase of the sensitivity.

\section{Conclusion}

Modalmetric sensor system can work successfully with different types of light sources, while maintaining its frequency characteristics. The parameter with the biggest direct impact on the work of modalmetric sensor is input optical power of the fiber circuit. Modalmetric sensor is able to generate greater amplitude of changes, only when the supplied power radiation is high enough. Modalmetric sensor in the single arm configuration has constant detection parameters in comparison with the transmission configuration and therefore it can be implemented. Moreover, by applying the modes scrambler to the structure of the fiber optic circuit, we can slightly improve the frequency response by making it more linear and improve sensitivity at the range of higher frequencies. In addition, modes scrambler can also reduce the length of the system circuit. Therefore, to develop the final system, authors recommend using the modes scrambler in order to stabilize operation of the laser output power as well as optical isolator in the form of optical circulator.

It should be noted that the authors were looking for a sensor which can be finally applied as a protection sensor of the museum collection. The obvious advantages of the medium is the response of frequency disorders as well as sensitivity. Fiber optic modalmetric sensor is a discrete system, thus it can be implemented into the structure of the protected object while remaining sensitive to mechanical disturbances (including the ability to react to breaking the fiber) more than a fiber optic interference sensors. It is characterized by linear response characteristics and bandwidth, limited only to the expected disturbance which significantly facilitate the operation on the commercial system. This fact can significantly reduce the final cost of the alarm sensor system.

\section{References}

[1] M. Życzkowski, Acta Phys. Pol. A 122, 933 (2012).

[2] M. Zyczkowski, B. Uziębło-Zyczkowska, L. Dziuda, K. Różanowski, Proc. SPIE 7894, 789404 (2011).

[3] Patent number: WO 2009/111820 A1; Future Fibre Technologies PTY LTD.

[4] M. Hasegawa, M. Kawahara, Proc. SPIE 8561, 856103 (2012).

[5] J.F. Wang, Y.X. Jin, Y. Zhao, X.-Y. Dong, Int. J. Light Electron Opt. 124, 1845 (2013).

[6] J.H. Wo, Q.Z. Sun, H. Liu, XL. Li, J.J. Zhang, D.M. Liua, P.P. Shum, Opt. Fiber Technol. 19, 289 (2013).

[7] J.W. Goodman, J. Opt. Soc. Am. 66, 1145 (1976).

Fig. 9. Characteristics of sensitivity as a function of frequency for different light sources, with mode scrambler. 